\title{
A simulation of key aspects of a primary process in natural photosynthesis by a Langmuir-Blodgett film assembly
}

\author{
Masaru Sakomura, ${ }^{* a}$ Kazuyoshi Ueda, ${ }^{a}$ and Masamichi Fujihira ${ }^{b}$ \\ ${ }^{a}$ Department of Material Science, Yokohama National University, Tokiwadai, \\ Hodogaya-ku, Yokohama 240-8501, Japan. Fax: +81 (45) 339-3971; Tel: +81 (45) \\ 339-3946; E-mail: kom@ynu.ac.jp \\ ${ }^{b}$ Department of Biomolecular Engineering, Tokyo Institute of Technology, Nagatsuta, \\ Midori-ku, Yokohama 226-8501, Japan. Fax: +81 (45) 924-5817; Tel: +81 (45) 924-5784; \\ E-mail: mfujihir@bio.titech.ac.jp
}

\begin{abstract}
An advanced Langmuir-Blodgett (LB) assembly designed to replicate the three key aspects (antenna, reaction center, and quinone pool) of a primary process in natural photosynthesis was successfully fabricated by alternate deposition of a mixed monolayer of an artificial reaction center and an antenna pigment and a pure bilayer of a second donor.
\end{abstract}

The development of an artificial photosynthesis system for the photovoltaic conversion of solar energy is one of the most fascinating challenges to respond to growing demand for sustainable energy supply. ${ }^{1}$ In our approach to design of photoelectric components with molecular dimensions for artificial photosynthesis, we had synthesized amphiphilic acceptor-sensitizer-donor (A-S-D) type triad molecules as models for a photosynthetic reaction center. $^{2}$ In a previous study, we had successfully observed the light-harvesting and the succeeding charge-separation on a monomolecular layer assembly including a triad and a light-harvesting antenna (ASD and $\mathrm{H}$, respectively, in Scheme 1). The fluorescence band of the pyrene moiety of $\mathrm{H}$ overlaps very well with the UV-vis absorption band of the acylated perylene $\mathrm{S}$ moiety of $\mathrm{ASD},{ }^{3}$ therefore the efficient energy transfer from photo-excited pyrene $\left(\mathrm{H}^{*}\right)$ to $\mathrm{S}$ can be expected to result.

In this report, we present herein a further advanced LB assembly as a biomimetic model system to seek to mimic the three successive aspects of photosynthesis: antenna pigment, reaction center, and quinone pool. In natural photosynthesis systems of higher plants, a lipid soluble and diffusible quinone called plastoquinone (PQ) acts as a mobile component of the electron

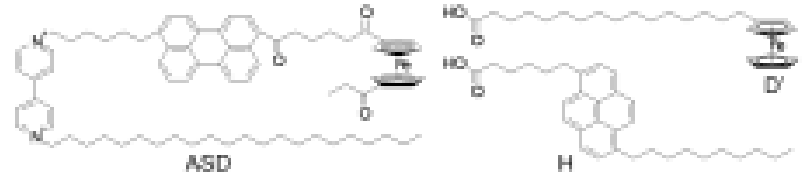

Scheme 1 Structural formulae of amphiphilic compounds.

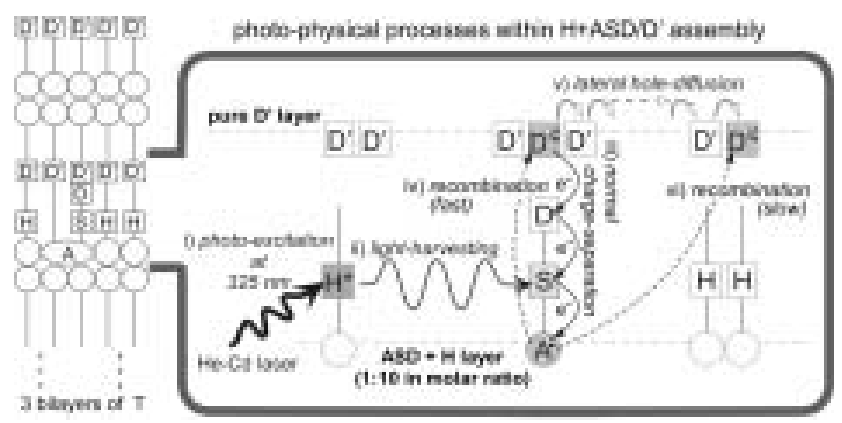
transport between two different photosynthetic reaction centers called photosystem I (PSI) and photosystem II (PSII). Not only the electron link but also an electron buffer in the form of a pool is a unique function of PQ. The electrons from PSII can be accumulated within the plastoquinone pool in the reduced form of plastoquinone, i.e., plastoquinol $\left(\mathrm{PQH}_{2}\right)$.

The surfactants shown in Scheme 1 were used for fabrication of the LB assembly studied in this report. The film structure and the expected photo-physical processes (i) - (vi) within the film are schematically illustrated in Fig. 1. A mixed monolayer of $\mathrm{H}$ and ASD and a pure bilayer of $\mathrm{D}^{\prime}$ were alternately deposited on a gold optically semi-transparent electrode (AuOTE) pre-coated with 3 bilayers of 22-tricosenoic acid (T). The transfer ratios of these films were almost unity, indicating ideal transfers. The thickness and structural uniformity of the films were confirmed by AFM. Based on our previous study, in which the light-harvesting by $\mathrm{H}$ was examined in acyl perylene-pyrene mixed films with a variety of mixing ratios, ${ }^{3}$ ASD in the mixed monolayer was diluted to the ratio of 1:10 with $\mathrm{H}$. The compound $\mathrm{D}^{\prime}$ was used as the second donor in this case. The ionization potential of the donor moiety of this compound is smaller than that of the $\mathrm{D}$ moiety of the triad, ${ }^{4}$ therefore the quadruplet system of A-S-D/D' was realized in the LB assembly. Photo-excitation of the $\mathrm{S}$ moiety will induce the charge-separation (iii) in the direction normal to the electrode surface. The resulting charge separated states can be detected as surface potential changes of the LB film by scanning surface potential microscopy (SSPM) ${ }^{5}$ The SSPM measurements were carried out in the same manner as described in former studies. ${ }^{6,7}$ A He-Cd laser with a CW output of $15 \mathrm{~mW}$ at $325 \mathrm{~nm}$ and $60 \mathrm{~mW}$ at $441.6 \mathrm{~nm}$ was used as an excitation light source. The two wavelengths were selected by the insertion of suitable band-pass and sharp-cut filters. In addition, neutral density filters were used for regulation of the light intensities. The powers of the incident beams reaching the surface of the AuOTE were estimated by a Si photodiode for precision photometry to be ca. $2.5 \times 10^{-3} \mathrm{~mW}$ at $325 \mathrm{~nm}$ and ca. $9.5 \times 10^{-3} \mathrm{~mW}$ at $441.6 \mathrm{~nm}$. 
Fig. 2(a) shows the change in the surface potential measured with the LB assembly under step illumination (125 ms) of $325 \mathrm{~nm}$ (solid curve) and $441.6 \mathrm{~nm}$ (dashed curve). As illustrated in Fig. 1, S* is induced by the light-harvesting (ii) from $\mathrm{H}^{*}$ to $\mathrm{S}$ under irradiation at $325 \mathrm{~nm}$, whereas $\mathrm{S}$ is directly excited at $441.6 \mathrm{~nm}$. The steep rise of both curves during the illumination time of $125 \mathrm{~ms}$ corresponds to the increase in the number of the charge separated species. In comparing these two curves, the peak intensity of the solid curve is ca. 2.5 times as large as that of the dashed curve. Considering the difference in the number of the incident photons estimated from the beam power and the photon energy, the 2.5 times increase in the peak intensity of the SSPM signal with the illumination at $325 \mathrm{~nm}$ corresponds to a 12.9 times increase in the charge-separation efficiency per incident photon. The significant increase in the charge-separation efficiency reflects the efficient light-harvesting in which the energy of photons absorbed by $\mathrm{H}$ at remote sites may migrate and be funnelled to the $\mathrm{S}$ moiety of ASD. ${ }^{3}$ The exponential-like decays with lifetimes of a few seconds after shutting the illumination down reflect the decreases in the number of the separated charges by recombination. However, the observed decays are too slow to be attributed to the charge recombination (iv) over the distance between A and D' moieties aligning in the direction normal to the electrode surface. ${ }^{8,9}$ The slow decay can be interpreted by assuming successive lateral hole-diffusion (v) via hole migration among neighbouring D' molecules.

So far such slow recombinations had been observed by SSPM on a variety of LB assemblies including triad and A-S type dyad monolayers, and explained by assuming intermolecular lateral electron- and hole-diffusion among the acceptor (A) and the donor (D or S) moieties, respectively. ${ }^{7,8}$ The accumulation of radical species with elapsed time of UV irradiation, detected by absorption measurements on LB assemblies including monolayers of A-S type dyads, had been also explained by the same mechanism of lateral charge-diffusion. ${ }^{10}$ Such lateral charge-diffusion via migration of holes and electrons between neighbouring donor and acceptor moieties, respectively, as assumed in these studies must be suppressed with the increase of the donor-to-donor and acceptor-to-acceptor distances. Hence, to confirm the lateral charge-diffusion mechanism, we had investigated the dependence of the SSPM signal on the dilution ratio of the mixed ASD monolayer with $T .^{7}$ In that study, the photo-response of the change in surface potentials had been observed clearly on the mixed LB monolayer with the molar ratio of ASD:T $=1: 5$, but never with the molar ratio of ASD:T $=1: 30$. Assuming ideal mixing of ASD and T in the mixed monolayers, ASD molecules are separated and isolated from one another with the latter dilution ratio. In the present case, ASD was diluted by $\mathrm{H}$ with molar ratio ASD:H = 1:10. The limiting area per molecule of $\mathrm{H}$ at an air-water interface is larger than that of $\mathrm{T}$, therefore the dilution of the ASD monolayer with $\mathrm{H}$ would be more effective in suppressing the lateral charge-diffusion than that with $\mathrm{T}$.

Fig. 2(b) shows the SSPM signal observed on a reference film in which, instead of the D' bilayer, a T bilayer was deposited on the mixed monolayer of ASD and H. The photo-response of the change in the surface potential induced by the step illumination at $441.6 \mathrm{~nm}$, with which the $\mathrm{S}$ moiety is directly excited but $\mathrm{H}$ contributes just a diluent, was surely observed, but the decay feature of the curve was rather obscured by the poor S/N ratio as represented by the dashed curve. On the other hand, the SSPM signal at $325 \mathrm{~nm}$ could be clearly observed as represented by the solid curve in Fig. 2(b). These results suggest that the lateral diffusion of the photo-produced anion and cation radicals among neighbouring A and D moieties, respectively, within the mixed monolayer of ASD and $\mathrm{H}$ are possible in the dilution condition ASD:H = 1:10. However, in comparing Figs. 2(a) and (b), the yield of the accumulated charges, which is reflected in the intensity of the SSPM signal, within A and D planes of the reference film is much lower than that within A and D' planes of the H+ASD/D' type assembly. The photo-induced surface potential changes at both wavelengths increased about one order of magnitude over those of the reference film by the addition of the D' bilayer as indicated in Fig. 2(a). The significant increases in the surface potential are attributable to the efficient lateral hole-diffusion, which is due to the prolonged lifetime of the perpendicular charge-separation by the A-S-D/D' quadruplet system and due to the enhanced rate of the lateral hole migration by the close packing of the second donor moiety in the plane of the pure $\mathrm{D}^{\prime}$ layer. ${ }^{6}$ Thus the $\mathrm{D}^{\prime}$ layer plays a role of a charge pool in our artificial photosynthesis system in the same way as the quinone pool in natural photosynthesis.

Interestingly, it was found that the ratio between the peak intensities of the two SSPM signals represented by the solid and the dashed curves in Fig. 2(b) seems very similar to that in Fig. 2(a). If the photo-induced electric fields between the two parallel planes of A and D for the reference film and of A and D' for the H+ASD/D' type assembly are homogeneous and proportional to the number of the accumulated charges, the efficiencies of the light-harvesting process (ii) can be directly related to the intensity of the SSPM signals. Assuming the simple model of a parallel-plate capacitor, the antenna effect of the light-harvesting process (ii) within the mixed monolayer with the molar ratio ASD:H $=1: 10$ would not be changed by the addition of the D' layer.

In summary, we have presented the biomimetic model system in which light-harvesting, charge-separation, and charge-diffusion and accumulation functions have been successfully integrated by fabricating a H+ASD/D' type LB assembly. As a consequence of the cooperation of these functions, the efficiency of the photo-induced surface potential 
change on the H+ASD/D' assembly by photo-excitation of the light-harvesting antenna $(\mathrm{H})$ has increased about one order of magnitude over that on the reference film without the second donor $\left(\mathrm{D}^{\prime}\right)$.

\section{Notes and references}

1 B. O'Regan and M. Grätzel, Nature, 1991, 253, 737; M. Grätzel, Pure Appl. Chem., 2001, 73, 459; D. Gust, T. A. Moore, A. L. Moore, S-J. Lee, E. Bittersmann, D. K. Luttrull, A. A. Rehms, J. M. DeGraziano, X. C. Ma, F. Gao, R. E. Belford and T. T. Trier, Science, 1990, 248, 199.

2 M. Fujihira, M. Sakomura and T. Kamei, Thin Solid Films, 1989, 180, 43; M. Sakomura and M. Fujihira, Thin Solid Films, 1994, 243, 616; M. Sakomura, S. Lin, T. A. Moore, A. L. Moore, D. Gust and M. Fujihira, J. Phys. Chem. A, 2002, 106, 2218; G. Kodis, P. A. Liddell, L. de la Garza, P. C. Clausen, J. S. Lindsey, A. L. Moore, T. A. Moore and D. Gust, J. Phys. Chem. A, 2002, 106, 2036; H. Imahori, D. M. Guldi, K. Tamaki, Y. Yoshida, C. Luo, Y. Sakata, and S. Fukuzumi, J. Am. Chem. Soc., $2001,123,6617$.

3 M. Fujihira, M. Sakomura and R. Sawada, Mol. Cryst. Liq. Cryst., 1998, 322, 313.

4 T. Kondo, H. Yamada, K. Nishiyama, K. Suga and M. Fujihira, Thin Solid Films, 1989, 179, 463.

5 M. Fujihira, H. Kawate and M. Yasutake, Chem. Lett., 1992, 2223; H. Yokoyama, K. Sato, and T. Inoue, Mol. Electron. Bioelectron, $1992, \mathbf{3}, 79$

6 M. Sakomura, T. Oono and M. Fujihira, Thin Solid Films, 1998, 327-329, 708.

M. Sakomura, T. Oono, R. Sakon and M. Fujihira, Ultramicroscopy, 2002, 91, 215.

8 M. Fujihira, M. Sakomura, D. Aoki and A. Koike, Thin Solid Films, 1996, 273, 168.

E. G. Wilson, Thin Solid Films, 1996, 273, 1.

10 M. Sakomura and M. Fujihira, Chem. Lett., 1998, 701; M. Sakomura and M. Fujihira, Thin Solid Films, 1998, 327-329, 718. 